Supplementary material

\title{
Procyanidins-loaded complex coacervates for improved stability by self-crosslinking and calcium ions chelation
}

Shanshan Tie ${ }^{\text {abcd }}$, Xuedi Zhangabcd, Haitao Wangabcd, Yukun Songabcd, and Mingqian $\operatorname{Tan}^{\text {abd }}{ }^{*}$

${ }^{a}$ School of Food Science and Technology, Dalian Polytechnic University, Dalian 116034, P. R. China

${ }^{b}$ National Engineering Research Center of Seafood, Dalian 116034, P. R. China

'Engineering Research Center of Seafood of Ministry of Education of China, Dalian 116034, P. R. China

${ }^{d}$ Collaborative Innovation Center of Seafood Deep Processing, Dalian 116034, P. R. China

*To whom correspondence should be addressed. E-mail: mqtan@dlpu.edu.cn, ORCID: 0000000275350035. Qinggongyuan 1, Ganjingzi District, Dalian 116034, P. R. China 
Table S1. Theoretical and detected molecular weights (Mw) of procyanidins (PCs) by MALD1-TOF-MS

\begin{tabular}{cccc}
\hline $\mathrm{PC}_{\mathrm{C}, \mathrm{G}}$ & $\mathrm{G}$ & Theoretical Mw & Detected Mw \\
\hline $\mathrm{PC}_{2,(0-2)}$ & 0 & 578.0 & 595.6 \\
& 1 & 730.0 & 731.5 \\
$\mathrm{PC}_{3,(0-2)}$ & 2 & 882.0 & 882.7 \\
& 1 & 866.0 & 866.8 \\
$\mathrm{PC}_{4,(0-2)}$ & 2 & 1018.0 & 1018.6 \\
& 0 & 1170.0 & 1170.8 \\
& 1 & 1154.0 & 1154.8 \\
$\mathrm{PC}_{5,(0-2)}$ & 2 & 1306.0 & 1307.0 \\
& 0 & 1458.0 & 1459.5 \\
& 1 & 1442.0 & 1443.3 \\
& 2 & 1594.0 & 1595.5 \\
\hline
\end{tabular}

$\mathrm{C}$ and $\mathrm{G}$ represent the number of flavan-3-ol units and gallate esters, respectively. 
Table S2. Effects of different formulations on microcapsulation efficiency (ME) and microcapsulation yield (MY) of procyanidins (PCs).

\begin{tabular}{|c|c|c|c|}
\hline $\begin{array}{l}\text { PC Concentration } \\
(\mathrm{mg} / \mathrm{mL})\end{array}$ & $\begin{array}{c}\mathrm{Ca}^{2+} \text { Concentration } \\
(\mathrm{mg} / \mathrm{mL})\end{array}$ & $\operatorname{ME}(\%)$ & MY (\%) \\
\hline \multirow[t]{4}{*}{3.75} & 0.00 & $68.89 \pm 2.46^{\mathrm{g}}$ & $67.12 \pm 2.74^{\mathrm{ef}}$ \\
\hline & 0.24 & $77.41 \pm 1.26^{\mathrm{de}}$ & $79.34 \pm 3.21^{\mathrm{b}}$ \\
\hline & 0.48 & $70.03 \pm 1.59^{\mathrm{de}}$ & $74.83 \pm 2.33^{c}$ \\
\hline & 0.72 & $72.32 \pm 0.47^{\mathrm{f}}$ & $70.83 \pm 3.59^{\mathrm{def}}$ \\
\hline \multirow[t]{4}{*}{5.00} & 0.00 & $71.40 \pm 2.29^{\mathrm{fg}}$ & $64.82 \pm 1.35^{\mathrm{e}}$ \\
\hline & 0.24 & $78.22 \pm 2.29^{\mathrm{de}}$ & $79.45 \pm 1.10^{\mathrm{b}}$ \\
\hline & 0.48 & $77.57 \pm 0.56^{\mathrm{de}}$ & $78.98 \pm 1.15^{\mathrm{b}}$ \\
\hline & 0.72 & $78.90 \pm 0.65^{\mathrm{cd}}$ & $81.42 \pm 0.82^{b}$ \\
\hline \multirow[t]{4}{*}{6.25} & 0.00 & $75.90 \pm 1.01^{\mathrm{e}}$ & $60.02 \pm 3.96^{\mathrm{f}}$ \\
\hline & 0.24 & $81.19 \pm 1.47^{\mathrm{bc}}$ & $87.86 \pm 2.67^{\mathrm{a}}$ \\
\hline & 0.48 & $82.16 \pm 0.46^{\mathrm{b}}$ & $72.19 \pm 1.59^{\mathrm{cd}}$ \\
\hline & 0.72 & $84.60 \pm 0.94^{\mathrm{a}}$ & $70.38 \pm 1.77^{\mathrm{def}}$ \\
\hline
\end{tabular}




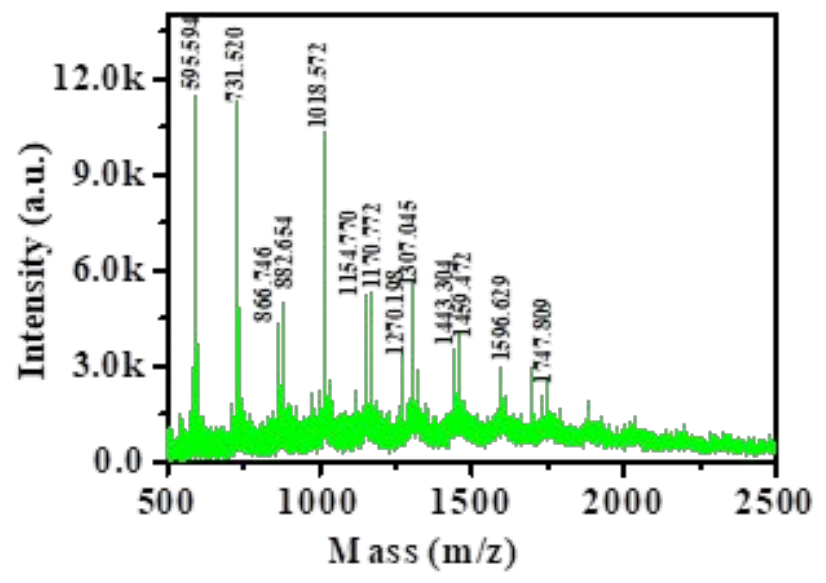

Figure S1. MALD1-TOF-MS spectrum of procyanidins (PCs) extracted from grape seeds. 


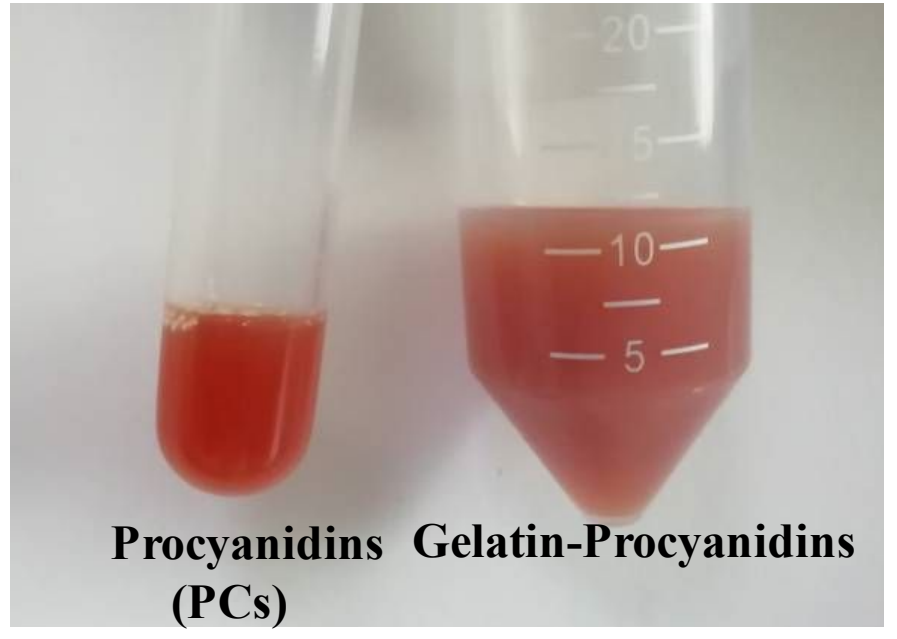

Figure S2. Photograph of procyanidins (PCs) and gelatin (GE)-PCs mixture in aqueous solution at $\mathrm{pH} 5.56$. 


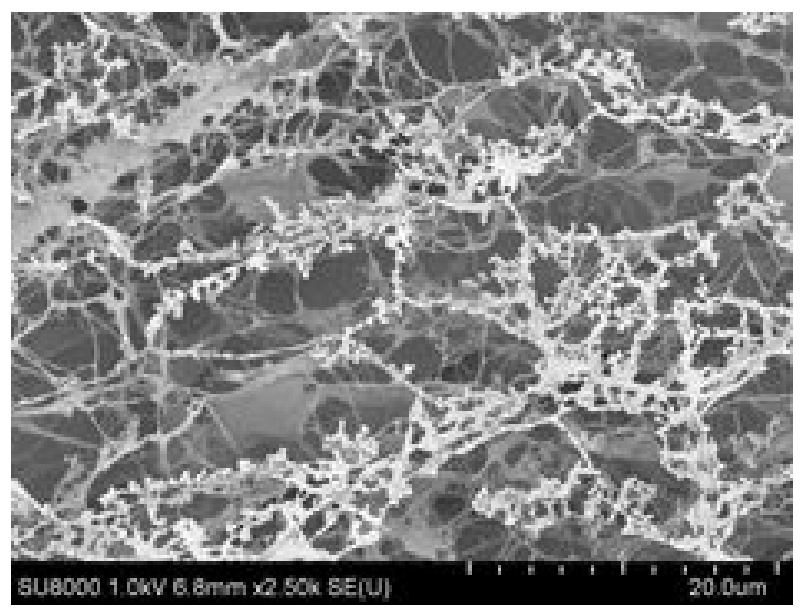

Figure S3. Scanning electron microscopy (SEM) image of sodium alginate (SA, only the polysaccharide) solution measured with a SU8010 field emission scanning electron microscope (FE-SEM, Hitachi, Tokyo, Japan) at an accelerating voltage of $1 \mathrm{kV}$. 